\title{
Prostate Neoplasm
}

National Cancer Institute

\section{Source}

National Cancer Institute. Prostate Neoplasm. NCI Thesaurus. Code C3343.

A benign, borderline, or malignant neoplasm that affects the prostate gland. Representative examples include benign prostate phyllodes tumor, prostatic intraepithelial neoplasia, prostate carcinoma, and prostate sarcoma. 